\title{
Thermal Conductivity and Heat Capacity of a Nylon- 6/Multi-wall Carbon Nanotube Composite Under Pressure
}

\author{
Junchun $\mathrm{Yu}^{\mathrm{a}}$, Bounphanh Tonpheng ${ }^{\mathrm{a}}$ and Ove Andersson ${ }^{\mathrm{a}}$ \\ ${ }^{a}$ Department of Physics, Umeå University, Umeå, Sweden
}

\begin{abstract}
The thermal conductivity, $\kappa$, of nylon-6 increased $22 \%$ whereas the heat capacity per unit volume, $\rho c_{p}$, decreased $10 \%$ by adding $2.1 \mathrm{wt} \%$ Multi-Wall Carbon Nanotubes MWCNTs. Simultaneously, the glass transition temperature, $T_{g}$, which was detected as a weak sigmoidal increase in $\rho c_{p}$ and a decrease in $\mathrm{d} \kappa / \mathrm{d} T$, increased $11 \mathrm{~K}$. These results show that the MWCNTs-nylon-6 interaction restricts the segmental mobility of nylon- 6 and decreases $c_{p}$ of nylon6.
\end{abstract}

Keywords: Thermal conductivity, heat capacity, nylon-6, multi-wall carbon nanotube, high pressure, glass transition. PACS: 62.50.-p, 65.60.+a, 81.05.Lg, 81.07.De

\section{INTRODUCTION}

Carbon nanotubes (CNTs) attract intensive attention because of their extraordinary thermal and mechanical properties, and high electrical conductivity. This new form of carbon material has great potential to be utilized in thermal management, high performance composites, electronics etc. [1]. The thermal conductivity $\kappa$ of single-wall carbon nanotubes (SWCNTs) and multi-wall carbon nanotubes (MWCNTs) has been studied experimentally. Fujii et al.[2] measured $\kappa$ of individual SWCNT and found $\kappa>2000 \mathrm{~W} \mathrm{~m}^{-1} \mathrm{~K}^{-1}$ for a CNT diameter of $9.8 \mathrm{~nm}$, which further increased with decreasing diameter. Kim et al.[3] reported $\kappa>3000 \mathrm{~W} \mathrm{~m}^{-1} \mathrm{~K}^{-1}$ for MWCNT at room temperature while that of SWCNTs and MWCNTs in bundles was $\sim 250$ and $\sim 20 \mathrm{~W} \mathrm{~m}^{-1} \mathrm{~K}^{-1}$ respectively $[4,5]$. Thus, adding relatively small amount of CNTs may dramatically improve $\kappa$ of poor thermal conductors such as polymers. Tonpheng et al. [6] reported an increase of $\kappa$ by $120 \%$ for polyisoprene with $5 \mathrm{wt} \%$ SWCNTs. The improvement was roughly proportional to the percentage of SWCNTs in the polymer composite, but the increase was lower than that predicted by simple mixture rules, which is likely due to high interfacial thermal resistance. In this work, we study $\kappa$, heat capacity and glass transition behavior for nylon- 6 and its MWCNT composite and also explore the possibility of improved interfacial contact and percolation through high-pressure densification.

\section{EXPERIMENTAL SECTION}

MWCNTs (Nanocyl-3150) were purchased from Nanocyl, Belgium and purified before use. A typical purification procedure included oxidation in air, washing in $37 \% \mathrm{HCl}$ acid (Sigma-Aldrich, ACS reagent) and, subsequently, rinsing by water, filtration and drying [7]. The synthesizes of the nylon-6/MWCNTs composite was done by in-situ polymerization [8], using sonication to disperse MWCNTs $(0.24 \mathrm{~g}$ or $2.1 \mathrm{wt} \%)$ in melted $\varepsilon$ caprolactam $(10 \mathrm{~g}$, Aldrich, $99 \%)$ at $80{ }^{\circ} \mathrm{C}$, adding 6-aminocaproic acid (1 g, Sigma, $\left.\geq 99 \%\right)$ as initiator afterwards and heating to $250{ }^{\circ} \mathrm{C}$ with simultaneous mechanical stirring under nitrogen atmosphere for $6 \mathrm{~h}$. A pure nylon- 6 control sample was synthesized using the same procedure.

The nylon-6/MWCNT and nylon-6 samples were hot pressed into plates (diameter: $\sim 37 \mathrm{~mm}$, thickness: $\sim 3 \mathrm{~mm}$ ) at $250^{\circ} \mathrm{C}$ in an argon atmosphere, and inserted in a custom made Teflon ${ }^{\circledR}$ cell. The Teflon ${ }^{\circledR}$ cell, contained a type $\mathrm{K}$ thermocouple and a hot-wire probe ( $0.1 \mathrm{~mm} \mathrm{Ni}$-wire) for measurements of $\kappa$ and $\rho c_{p}$ using the hot-wire method [9]. The cell was mounted in a piston cylinder apparatus and later loaded in a hydraulic press. The sample could be 
heated up to ca. $530 \mathrm{~K}$ by an electric heater surrounding the cylinder and cooled down to ca. $100 \mathrm{~K}$ by liquid nitrogen. The estimated inaccuracies are $\pm 2 \%$ in $\kappa$ and $\pm 5 \%$ in $\rho c_{p}$.

Transmission electron microscope (TEM, JEM-1230) and tapping mode Atomic Force Microscopy (AFM, MultiMode, Veeco Metrology) were performed to exam the MWCNTs purity and the dispersion of MWCNTs in nylon-6. Differential scanning calorimetry (DSC, Pyris Diamond) was used to analyze the degree of crystallinity.

\section{RESULTS AND DISCUSSION}

As shown in Figure 1, height and amplitude images of the $2.1 \mathrm{wt} \%$ nylon-6/MWCNT composite suggest a fairly good dispersion of MWCNTs in the nylon-6 matrix. The long, tubular and twisted structures, which are the polymer wrapped MWCNTs, have a typical diameter of $\sim 50 \mathrm{~nm}$. The TEM image (insert) shows that highly purified MWCNTs, with no visible amounts of amorphous carbon or metallic catalyst, are obtained after purification.

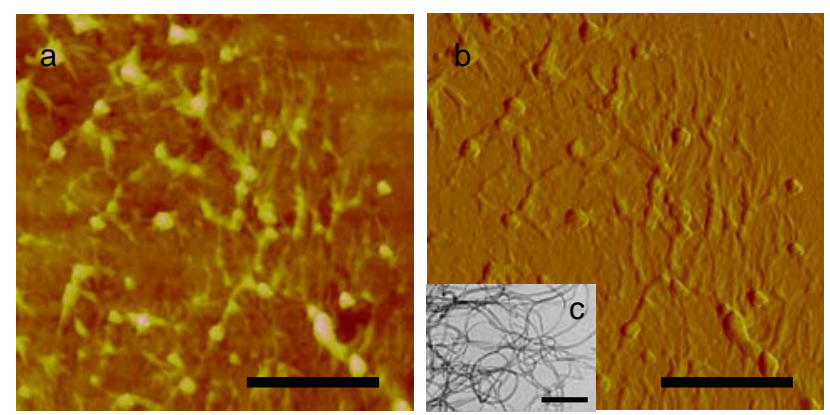

FIGURE 1. AFM image of Nylon-6/MWCNTs composite: (a) height image, (b) amplitude image (scale bar is $1 \mu \mathrm{m}$ ). The insert (c) is a TEM image of the purified MWCNTs (scale bar is $200 \mathrm{~nm}$ )

A pressure scan of both nylon- 6 and its composites was performed at $298 \mathrm{~K}$ from low pressure to $1.0 \mathrm{GPa}$, as indicated in Figure 2a. The thermal conductivity of both samples increases due to densification, which reduces the inter-particle distances and, thus, improves the heat transfer between polymer chains. However, there is no indication of a stronger increase for the composite, which shows that the densification is insufficient to form a percolation network of the MWCNTs and that the thermal contact between the MWCNTs and nylon- 6 does not improve sufficiently to make an impact on $\kappa(p)$. The behavior of $\kappa$ below $1 \mathrm{GPa}$ is therefore mainly governed by the changes in the nylon-6 matrix.

Figure $2 \mathrm{~b}$ shows $\kappa(T)$ from 290 to $440 \mathrm{~K}$ at $0.8 \mathrm{GPa}$ for both nylon-6 and the $2.1 \mathrm{wt} \%$ nylon-6/MWCNT composite. The thermal conductivity of nylon- 6 shows a distinct change in $\mathrm{d} \kappa / \mathrm{d} T$ from positive to negative near 368 $\mathrm{K}$, which is a typical behavior at the glass transitions temperature $T_{g}$ of polymers [10]. This result is also confirmed by the simultaneously measured data for $\rho c_{p}$ (figure 2c). These show the (stretched weak) sigmoidal increase, which is characteristic of a glass transition. Thus, $T_{g}$ of nylon-6, which is difficult to observe in several other characteristics that normally show clear $T_{g}$ features, gives a distinct change in the temperature dependence of $\kappa$. The corresponding results for the composite are similar but with a change in $\mathrm{d} \kappa / \mathrm{d} T$ at $379 \mathrm{~K}$, i.e. the $2.1 \mathrm{wt} \%$ MWCNTs raised $T_{g}$ about $11 \mathrm{~K}$. The shift of $T_{g}$ is due to restricted segmental mobility, which is apparently caused by the MWCNT-nylon-6 interaction or, in terms of the free volume theory, the MWCNTs reduces the free volume or voids in the polymer.

The $\kappa$ of the composite is $0.549 \mathrm{~W} \mathrm{~m}^{-1} \mathrm{~K}^{-1}$, which is $\sim 22 \%$ higher than that of nylon- $6\left(0.452 \mathrm{~W} \mathrm{~m}^{-1} \mathrm{~K}^{-1}\right)$ at 0.8 $\mathrm{GPa}, 298 \mathrm{~K}$. As shown in Table 1, the degree of crystallinity of nylon-6 and the composite was the same, $\sim 20 \%$ (melting enthalpy, $\Delta H$, of $100 \% \alpha$ crystalline nylon-6 is $241 \mathrm{~J} \mathrm{~g}^{-1}$ ) [11], which shows that the enhanced $\kappa$ of the composite was caused by the high $\kappa$ of the MWCNTs and not by a change of crystallinity. Another interesting observation is the significant MWCNT induced decrease of $\rho c_{p}$ from $1.19 \mathrm{MJ} \mathrm{m}^{-3} \mathrm{~K}^{-1}$ for nylon-6 to $1.07 \mathrm{MJ} \mathrm{m}^{-3} \mathrm{~K}^{-1}$ for the composite at atmosphere pressure and $298 \mathrm{~K}$ (not shown in figure), i.e. a decrease of $10 \%$. Since this is significantly larger than $0.2 \%$ calculated by the mixture rule $[6,12]$, it means that the MWCNTs decreases the heat capacity of nylon-6. Moreover, since the relative decrease is about the same above $T_{g}$ as that below (figure $2 \mathrm{c}$ ), the MWCNT- nylon-6 interaction apparently reduces the vibrational and configurational heat capacities of nylon-6 to about the same extent, and this effect should make the nylon-6 matrix slightly more rigid.

TABLE 1. Melting enthalpy $(\Delta H)$ and crystallinity $\left(C_{D S C}\right)$ determined by DSC

\begin{tabular}{lcc}
\hline Sample & $\boldsymbol{\Delta H}(\mathbf{J} / \mathbf{g})$ & $\boldsymbol{C}_{\boldsymbol{D S C}}$ \\
\hline Virgin Nylon-6 & 47.9 & $19.9 \%$ \\
$2.1 \mathrm{wt} \%$ MWCNTs in nylon-6 & 47.4 & $19.7 \%$ \\
\hline
\end{tabular}



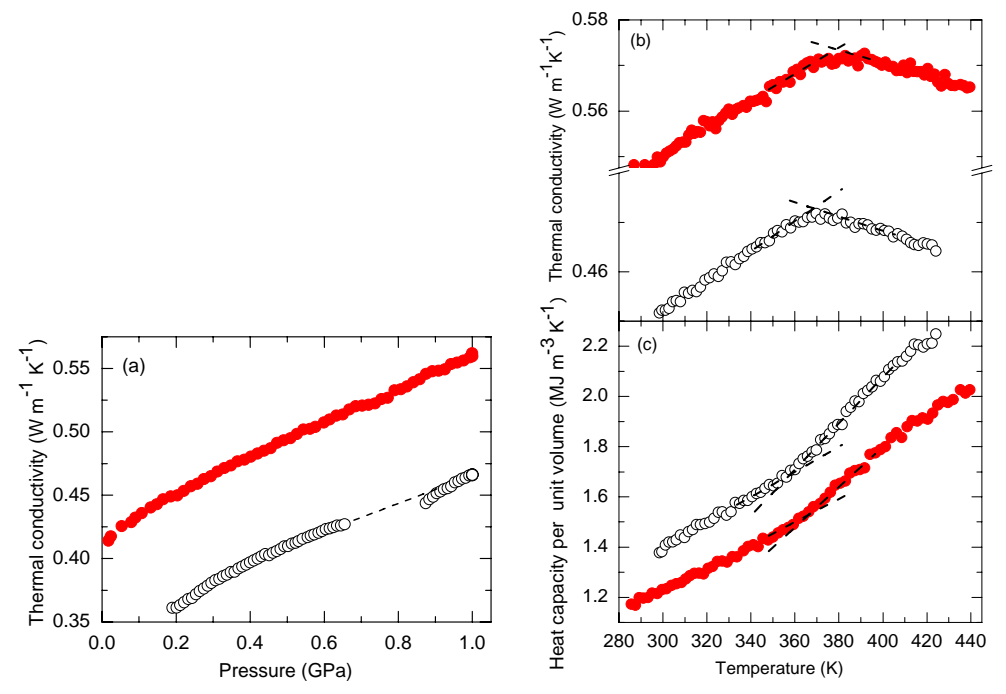

FIGURE 2. (a)Thermal conductivity for: $(\bullet) 2.1 \mathrm{wt} \%$ nylon-6/MWCNT composite and (०) nylon-6 measured on increasing pressure at $298 \mathrm{~K}$. The dashed line represents a range where the data for nylon- 6 were affected by a transition in the surrounding Teflon. (b) Thermal conductivity and (c) heat capacity per unit volume of: $(\bullet) 2.1 \mathrm{wt} \%$ nylon-6/MWCNT composite and (०) nylon- 6 at $0.8 \mathrm{GPa}$ measured on heating. The dash lines in both panels represent linear functions fitted to the data below and above the distinct change of slope. The point of intersection represents $T_{g}$.

\section{CONCLUSIONS}

In summary, $\kappa$ and $\rho c_{p}$ for nylon- 6 and its $2.1 \mathrm{wt} \%$ MWCNT composite have been measured, in-situ, under high pressure by the transient hot-wire method. The high $\kappa$ of the MWCNTs increased $\kappa$ of the composite by $22 \%$. This increase was almost independent of pressure, which shows that the densification was insufficient to cause thermal percolation through the MWCNTs. Simultaneously, the MWCNTs increased $T_{g}(11 \mathrm{~K})$ but decreased $\rho c_{p}(10 \%)$ of nylon-6, which reveal MWCNT induced effects on the bulk properties of the nylon-6 matrix. These effects may have a similar origin and seems to be associated with the MWCNT-nylon-6 interfacial interaction.

\section{ACKNOWLEDGMENTS}

This work was financially supported by Swedish Research Council.

\section{REFERENCES}

1. M. Moniruzzaman and K. I. Winey, Macromolecules 39 (16), 5194-5205 (2006).

2. M. Fujii, X. Zhang, H. Xie, H. Ago, K. Takahashi, T. Ikuta, H. Abe and T. Shimizu, Phys. Rev. Lett. 95 (6), 065502 (2005).

3. P. Kim, L. Shi, A. Majumdar and P. L. McEuen, Phys. Rev. Lett. 87 (21), 215502 (2001).

4. W. Yi, L. Lu, Z. Dian-lin, Z. W. Pan and S. S. Xie, Physical Review B 59 (14), R9015 (1999).

5. D. J. Yang, Q. Zhang, G. Chen, S. F. Yoon, J. Ahn, S. G. Wang, Q. Zhou, Q. Wang and J. Q. Li, Physical Review B 66 (16), 165440 (2002)

6. B. Tonpheng, J. Yu and O. Andersson, Macromolecules 42 (23), 9295-9301 (2009).

7. J.-M. Moon, K. H. An, Y. H. Lee, Y. S. Park, D. J. Bae and G.-S. Park, The Journal of Physical Chemistry B 105 (24), $5677-$ 5681 (2001).

8. J. Gao, M. E. Itkis, A. Yu, E. Bekyarova, B. Zhao and R. C. Haddon, Journal of the American Chemical Society 127 (11), 3847-3854 (2005).

9. H. Bjorn and G. R. Russell, Journal of Applied Physics 68 (7), 3285-3292 (1990).

10. D. W. Van Krevelen, Properties of polymers: correlations with chemical structure. (Elsevier publishing company, 1972).

11. V. K.-H. Illers and H. Haberkorn, Die Makromolekulare Chemie 142 (1), 31-67 (1971).

12. N. R. Pradhan, H. Duan, J. Liang and G. S. Iannacchione, Nanotechnology 20 (24), 7 (2009). 